\title{
No compromise in correlative microscopy: One sample, one preparation protocol for CLSM and TEM
}

\author{
S.S. Biel, K. Kawaschinski, K.P. Wittern, U. Hintze, R. Wepf \\ Beiersdorf AG, Analytical Microscopy Dept., 20245 Hamburg, Germany
}

Structural investigation of tissue biopsies requires the coupling of optimal structural sample preservation with detailed information collected at the light and electron microscopic level. Unfortunately, although cryo-immobilisation by high-pressure freezing provides the best structural preservation, it is used routinely only for EM investigations, while for light microscopy chemical fixation protocols have been established. These chemically invasive fixation protocols have the drawback of introducing unpredictable fixation artefacts. Therefore, comparative histopathological (i.e. light microscopic) and ultrastructural (i.e. electron microscopic, EM) results are usually obtained from parallel samples that have not been prepared identically and never by examining exactly the same features in exactly the same, optimally preserved sample. Finally, finding an area of interest for EM investigation within a complex tissue is like searching for a needle in a haystack.

To overcome these handicaps, we modified the well-established freeze-substitution technique (FS), to allow us to investigate resin-embedded cryo-immobilised tissue by confocal laser scanning microscopy (CLSM) prior to EM examination. Thus, (1) selected cells throughout the whole tissue block can be depicted by CLSM and subsequently (2) selectively prepared by targeted sectioning for follow-up investigation of the identical structure by TEM. This is facilitated by the addition of specific fluorescent dyes during the first FS exchange step. Selective binding properties of various dyes to different cellular structures allow a direct histological description of the tissue at the light microscope level. After embedding and preparation of a blockface, the specimen can first be examined by CLSM. For areas of interest, the depth in the resin block is determined followed by removal of the tissue lying above. Then, the cell layer can be cut into a series of ultrathin sections and examined by EM for determination of the sub-cellular and nano-structural organisation. 


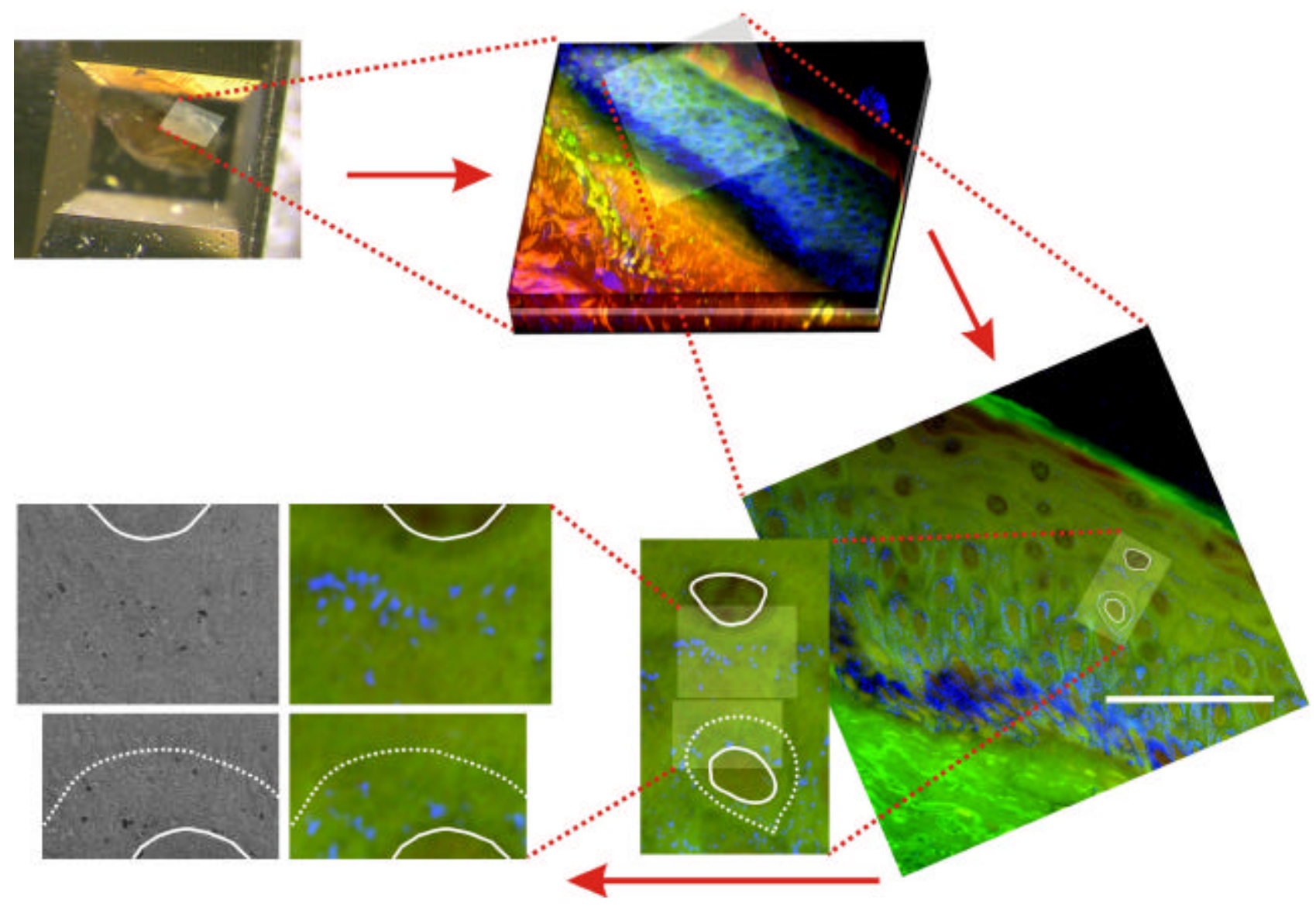

\section{Correlating light and electron microscopy: the process.}

The starting point of the correlative microscopy procedure is the plain blockface in the upper lefthand corner. The sample is freeze-substituted in the presence of acridine orange and afterwards embedded in Epon. From this blockface, a stack of confocal images is collected (Step 1). After evaluating the 3D data, an area of interest is chosen within a single 2D image and the depth within the resin block is determined (Step 2). Ultrathin sections are taken at the corresponding depth and investigated by TEM (Step 3). Finally, the TEM information is correlated with the confocal images, e.g. nuclei (solid line) or cell borders (dotted line). Scale bar: $\mathbf{5 0} \boldsymbol{\mu m}$ 